\title{
NOTE SUR LES DRACUNCULIDES D'OISEAUX
}

\section{Par Alain-G. CHABAUD, Yvonne CAMPANA et TRUONG-TAN-NGOC}

L'un de nous a souligné en 1937 l'importance économique d'un Dracunculide, parasite du canard domestique en Extrême-Orient. La femelle du parasite, lorsqu'elle est mature, forme des tumeurs situées généralement sous le menton, plus rarement aux membres inférieurs, et elle perce la peau pour émettre ses larves, en créant des désordres plus ou moins graves chez l'hôte.

L'examen de ces Nématodes, pratiqué en 1937 au Laboratoire de Parasitologie de l'Institut de Médecine coloniale de Marseille, avait permis d'assimiler cette espèce à Oshimaia taiwana (Sugimoto 1919).

En 1934, l'année même où Sugimoto créait le nouveau genre Oshimaia, rangé dans la famille des Philometridæ, Wehr et Chitwood en Amérique créaient de leur côté, dans la famille des Dracunculidæ, la sous-famille des Avioserpensinæ pour le nouveau genre Avioserpens.

Ayant eu l'occasion d'étudier un Nématode du gen̨re Avioserpens récolté en France, nous avons été heureux d'avoir quelques exemplaires d'Oshimaia pour pouvoir préciser les rapports existant entre les deux genres.

Matériel. - Six tumeurs fibreuses prélevées dans la région sousmandibulaire de canards domestiques à Cholon (Sud Viet-Nam) le 10 novembre 1949. Ces pièces ont malheureusement été conservées in toto dans le formol et l'isolement des Nématodes pelotonnés à l'intérieur est très difficile. Il a été possible d'extraire deux fragments longs de $19,5 \mathrm{~cm}$., sept extrémités postérieures et quatre extrémités antérieures. Aucun mâle n'a été trouvé dans ces tumeurs. Les fragments de femelles ne sont pas dans un état de conservation suffisant pour en faire une étude détaillée, mais ils permettent cependant d'établir les caractères suivants :

Description. - Corps long de plus de $19 \mathrm{~cm}$. et large de $750 \mu$. L'extrémité antérieure est arrondie et n'est séparée du corps par

Annales de Parasitologie, T. XXV, $\mathrm{N}^{\circ} 4,-1950$. 
aucune constriction. Le bouclien céphalique est constitué par une cuticule épaisse formant une calotte nettement limitée. La tête en vue apicale est exactement superposable à celle d'Avioserpens galliardi que nous avons décrit récemment (1949), et nous renvoyons à cette publication pour la description et les figures.

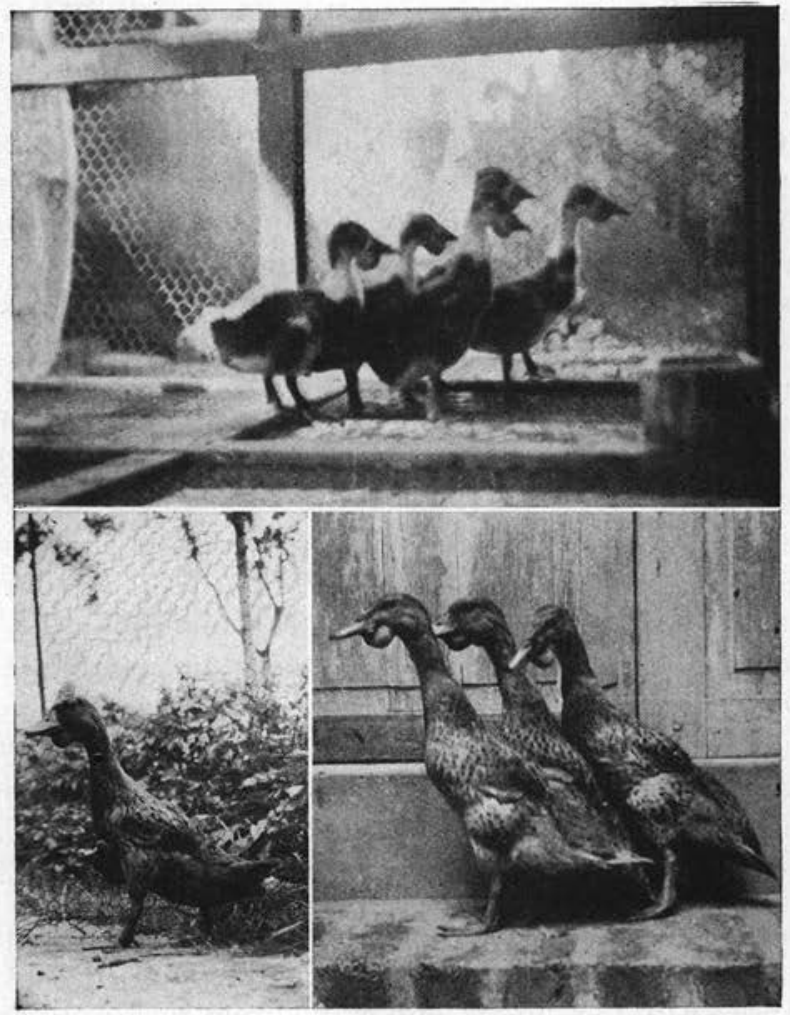

Canards domestiques d'âges variés, porteurs de tumeurs sous-mandibulaires à Avioserpens taiwana.

L'anatomie de l'œesophage est analogue à celle des autres Dracunculides : osophage musculaire long de $325 \mu$; œsophage glandulaire long de 2,5 mm., élargi dans sa partie antérieure en une masse globuleuse longue de $685 \mu$ et large de $490 \mu$. L'anneau nerveux est situé sous cet amas glandulaire à $1,2 \mathrm{~mm}$. de l'apex. Vulve impossible à déceler sur ces exemplaires matures. L'utérus remplit pres- 
que entièrement la cavité générale et se poursuit par un oviducte et un ovaire courts $(2-3 \mathrm{~mm}$.) à chaque extrémité.

Extrémité postérieure fine terminée par un petit mucron. Le rectum et l'anus sont atrophiques et la place de l'anus est très variable, éloignée de $1,5 \mathrm{~mm}$. à plus de $2,5 \mathrm{~mm}$. de la pointe de la queue. La forme générale de la queue est assez constante ; elle décrit une large courbe à concavité dorsale, mais l'extrême pointe au contraire se courbe ventralement. Les embryons intra-utérins sont identiques à ceux de tous les Dracunculides et mesurent en moyenne $420 \mu$.

Discussion. - Tous les caractères précédents répondent à la définition du genre Avioserpens.

Les différences qui existent avec la définition du genre Oshimaia proviennent d'erreurs d'observations, qui sont parfois difficiles à éviter avec un matériel aussi délicat :

$1^{\circ}$ Sugimoto n'ayant vu ni les amphides, ni les diérides, ni le pore excréteur a pris les faces dorsales et ventrales pour les faces latérales. Les grandes papilles qu'il a remarquées sur les bords de la bouche ne sont donc pas latérales; ce sont les papilles médianes ventrales et dorsales. Elles sont surmontées d'un dôme transparent à pointe bifide qui paraît être très caractéristique du genre Avioserpens.

$2^{\circ}$ Il n’y a pas d'anneau péribuccal bien défini comme dans le genre Dracunculus.

$3^{\circ}$ La figure publiée par Sugimoto (pl. XVII, fig. 3) montre que la dilatation de la glande œsophagienne dorsale a été confondue avec l'anneau nerveux. L'anneau nerveux est en réalité situé en arrière de cette masse glandulaire ; l'erreur ainsi commise provient du fait que la dilatation œsophagienne relativement peu opaque chez certains spécimens permet de voir la lumière œsophagienne par transparence. Cet amas glandulaire existe cependant de facon constante et rattache ces Nématodes à la famille des Dracunculidæ et non à celle des Philometridæ.

$4^{\circ}$ Wehr et Chitwood ont ajouté à leur diagnose générique : "Female with conical tail bent dorsad». Nous avons vu qu'il existe chez A. taiwana une double courbure, d'abord dorsale, ensuite ventrale ; cette forme se retrouve exactement sur la figure publiée par Singh (1949) pour A. multipapillosa. La queue de A. galliardi est à peu près rectiligne, avec l'extrême pointe courbée ventralement. Le caractère «queue courbée dorsalement » doit done disparaître de la diagnose du genre Avioserpens. 
En conclusion, il n'existe pas la moindre différence entre les genres Avioserpens et Oshimaia et ce dernier nom doit tomber en synonymie, puisque la publication de Wehr et Chitwood est antérieure de quelques mois.

Il existe actuellement quatre espèces d'Avioserpens':

a. A. taiwana (Sugimoto 1919) en Extrême-Orient.

b. A. denticulophasma Wehr et Chitwood 1934 en Amérique du Nord.

c. A. galliardi Chabaud et Campana 1949 en France.

d. A. multipapillosa Singh 1949 aux Indes.

La description d'A. denticulophasma étant extrêmement brève st ne comportant que très peu de données numériques ne permet pas de distinguer l'espèce d'A. taiwana.

La femelle d'A. galliardi, en dehors d'un œesophage particulièrement long, ne présente aucune différence nette avec $A$. taiwana. La longueur des embryons, différente de celle de A. denticulophasma, ne semble pas être un bon caractère spécifique, car Sugimoto (1934) constate de grandes variations de taille suivant que les mensurations sont faites sur des larves intra-utérines ou sur des larves libres.

A. multipapillosa n'a pas non plus de caractères particuliers et nous ne croyons pas que les deux critères donnés par l'auteur pour la distinguer de $A$. denticulophasma aient une valeur spécifique : d'une part, il est normal que les dimensions soient plus faibles chez un exemplaire non mature [voir à ce sujet les mensurations faites par Desportes, 1938, au cours du développement de Dracunculus besophagea (Polonio 1859) ] ; d'autre part, nous avons vu précédemment que la courbure de la queue était double (dorsale en avant, ventrale en arrière). Il n'est donc pas étonnant que les descriptions diffèrent un peu suivant l'exemplaire et suivant l'auteur.

En conclusion, il n'existe aucun caractère spécifique susceptible de différencier les femelles des quatre espèces d'Avioserpens déjà décrites.

Cependant, nous ne pensons pas qu'il y ait avantage actuellement à mettre toutes les espèces en synonymie avec $A$. taiwana, car, pour tous les Dracunculides, la diagnose se fait essentiellement d'après l'extrémité postérieure du mâle ; or, jusqu'à maintenant, seul le mâle d'A. galliardi a été décrit (1).

(1) Nous ne pouvons malheureusement pas préciser dans cette nouvelle note la morphologie du mâle d'A. galliardi, car en 1949 un héron cendré qui avait absorbé des Cyclops infestés expérimentalement, est resté négatif et nous n’avons pas pu nous procurer d'autre matériel depuis cette date. 
Les mâles sont difficiles à trouver, car il est probable qu'après la fécondation, ils n'accompagnent pas les femelles dans leurs migrations. Le moyen le plus efficace pour les récolter est d'abandonner pendant quelques heures le cadavre désárticulé de l'oiseau dans l'eau physiologique et d'examiner ensuite le produit de décantation.

\section{RÉsumé}

Oshimaia taiwana (Sugimoto 1919) est en réalité un Avioserpens. Les différences qui existent entre les définitions génériques des deux groupes sont dues uniquement à des erreurs d'interprétation et le genre Oshimaia tombe en synonymie.

Il existe donc quatre espèces d'Avioserpens, dont trois ne sont connues que par leur femelle. Aucun caractère spécifique ne permet de les différencier, mais il semble utile de conserver leur dénomination actuelle tant que les mâles n'auront pas été décrits.

\section{Bibliographie}

Chabaud (A.-G.) et Campana (Y.). - Avioserpens galliardi n. sp., parasite de l'aigrette Egretta garzetta, L. Ann. Parasit., XXIV, 1949, 67-76.

Despontes (C.). - Filaria asophagea Polonio 1859, parasite de la couleuvre d'Italie, est un Dracunculus très voisin de la filaire de Médine. Ann. Parasit., XVI, 1938, 305-326.

Singh (S. N.). - Studies on the Helminth Parasites of Birds in Hyderabad State. Nematoda, IV. J. Helminth., XXIII, 1949, 39-56.

Sugrmoto (M.). - List of zooparasites of the domestic Animals in Formose. Bull 133, Depart. Agric. Gouver. Res. Inst. Taihoku, 1919.

- On the Filaria from the Formosan domesticated Birds. J. Japan. Soc. Veter. Sc., XIII, déc, 1934, 261-266 + 3 pl.

Truong TAN Ngoc. - Filariose du canard domestique en Cochinchine due à Oshimaia taimana (Sugimoto 1919). Bull. Soc. Path. exot., XXX, 1937, 775-778.

Wehr (E. E.) et Chrrwoon (B. G.). - A new nematode from birds. Proc. Helm., Soc., I, mars 1934, 410-11.

Institut de Parasitologie de la Faculté de Médecine de Paris. (Directeur : Prof. H. Galliard). 